\title{
Detection of charge density wave ground state in granular thin films of blue bronze $\mathrm{K}_{0.3} \mathrm{MoO}_{3}$ by femtosecond spectroscopy
}

\author{
D. Dominko, ${ }^{1, a)}$ D. Starešinić, ${ }^{1}$ K. Salamon, ${ }^{1}$ K. Biljaković, ${ }^{1}$ A. Tomeljak, ${ }^{2,3}$ H. Schäfer, ${ }^{3}$ \\ Tim Huber, ${ }^{3}$ J. Demsar, ${ }^{2,3}$ G. Socol, ${ }^{4}$ C. Ristoscu, ${ }^{4}$ I. N. Mihailescu, ${ }_{2}^{4}$ Z. Siketić, ${ }^{5}$ \\ I. Bogdanović Radović, ${ }^{5}$ G. Pletikapić, ${ }^{5}$ V. Svetličić, ${ }^{5}$ M. Đekić, ${ }^{6}$ H. Samić, ${ }^{7}$ \\ and J. Marcus ${ }^{8}$ \\ ${ }^{1}$ Institute of Physics, HR-10001, Zagreb, P.O. Box 304, Croatia \\ ${ }^{2}$ J. Stefan Institute, Jamova 39, SI-1000, Ljubljana, Slovenia \\ ${ }^{3}$ Department of Physics and Center for Applied Photonics, University of Konstanz, D-78457 Konstanz, \\ Germany \\ ${ }^{4}$ Laser-Surface-Plasma Interactions Laboratory, Lasers Department, National Institute for Lasers, \\ Plasma and Radiation Physics, P.O. Box MG-54, Magurele, Ilfov, Romania \\ ${ }^{5}$ Ruđer Bošković Institute, Bijenička 54, HR-10001, Zagreb, Croatia \\ ${ }^{6}$ Faculty of Science, Zmaja od Bosne 33-35, Sarajevo, BA-71 000, Bosnia and Herzegovina \\ ${ }^{7}$ Faculty of Electrical Engineering, Zmaja od Bosne bb, Sarajevo, BA-71 000, Bosnia and Herzegovina \\ ${ }^{8}$ Institut Neel, CNRS, BP 166, F-38042, Grenoble, Cedex 9, France
}

(Received 6 April 2011; accepted 26 May 2011; published online 12 July 2011)

\begin{abstract}
During the last years, femtosecond time-resolved spectroscopy (fsTRS) has become an important new tool to investigate low energy excitations in strongly correlated systems. By studying energy relaxation pathways linking various degrees of freedom (e.g., electrons, spin, or lattice), the interaction strengths between different subsystems can be deduced. Here we report on yet another application of fsTRS, where the technique is used to unambiguously determine the nature of the ground state in granular thin films of a prototype charge density wave system blue bronze, $\mathrm{K}_{0.3} \mathrm{MoO}_{3}$. These, potassium blue bronze, films, obtained for the first time ever, have been prepared by pulsed laser deposition and investigated by various standard characterization methods. While the results of all used methods indicate that the thin films consist of nanometer grains of $\mathrm{K}_{0.3} \mathrm{MoO}_{3}$, it is only the non-destructive fsTRS that demonstrates the charge density wave nature of the ground state. Furthermore, the comparison of the fsTRS data obtained in thin films and in single crystals shows the reduction of the charge density wave transition temperature and of the photoinduced signal strength in granular thin films in respect to single crystals, which is attributed to the granularity and crystal growth morphology. (C) 2011 American Institute of Physics.
\end{abstract} [doi:10.1063/1.3606418]

\section{INTRODUCTION}

Low dimensional charge density wave (CDW) systems, with their inherently multi-component order parameter, present a prime example of cooperative phenomena in correlated electron systems. ${ }^{1}$ The CDW state is characterized by the periodic modulation of the electron density in the most conducting or linear chain direction coupled to the periodic lattice distortion corresponding to the macroscopically occupied "frozen-in" phonon mode.

In the thermodynamic limit, the CDW order parameter is given either by the lattice distortion or by the electron density modulation, as they are mutually proportional. However, when the electronic system is perturbed by a femtosecond optical pulse, the electronic and lattice parts of the order parameter become decoupled and show different dynamics. ${ }^{2,3}$ Probing the dynamics of the electronic and lattice system separately enables one to obtain information about the interplay between the electronic and lattice part of the order parameter, which is not possible by equilibrium spectroscopic methods.

a) Electronic address: ddominko@ifs.hr.
Indeed, systematic excitation intensity dependence studies of the phenomena have shown that the melting of the modulation of the electron density can be achieved on the time scale shorter than the characteristic lattice vibration period. This results in a state where the electronic system is in its high temperature metallic state, while the lattice is still in its low temperature modulated state. ${ }^{2}$ Consequently, the analysis of the temperature dependence of the low energy phonon spectra with the time dependent Ginzburg-Landau (TDGL) revealed that the CDW is in a non-adiabatic regime, where electrons do not adiabatically follow the lattice. ${ }^{3}$

The manifestation of this effect is the incomplete softening of the low temperature phonon (amplitudon) modes and the observation of an over-damped mode. The temperature dependence of the over-damped mode is consistent with its predominantly electronic character, describing the ultrafast recovery of the electronic modulation on the time-scale as much shorter than the lattice recovery time, a behavior that is expected within the framework of the TDGL model. ${ }^{3}$ The analysis of the femtosecond time-resolved spectroscopy (fsTRS) data with TDGL model suggests that all low frequency Raman active modes, which appear below the transition temperature from metallic to CDW state $\left(T_{\mathrm{p}}\right)$ are a result 
of linear coupling of the electronic part of the order parameter (EOP) to the $2 k_{\mathrm{F}}$ (CDW modulation wave-vector) phonon modes of the high temperature phase. Importantly, the TDGL model further suggests that linear coupling of the phonon modes with EOP initiates both Raman active modes, observed by time-resolved optical spectroscopy, ${ }^{3}$ as well as infrared (IR) active modes, as seen by infrared spectroscopy. ${ }^{4}$

In order to study the real-time dynamics of IR active phonon modes, as well as the (IR active) phase modes of the $\mathrm{CDW}$ in the $\mathrm{THz}$ frequency range, ${ }^{5}$ it would be beneficial to have CDW samples in a form of large area thin films, which would allow time-resolved $\mathrm{THz}$ conductivity dynamics studies to be performed in transmission geometry. ${ }^{6-9}$ Moreover, in pump-probe measurements the effect of steady state heating (built-up heating that is equivalent to heating due to illumination with continuous laser of the same average power) of the sample is quite pronounced. In particular, in samples with low thermal conductivity, such as $\mathrm{K}_{0.3} \mathrm{MoO}_{3},{ }^{10}$ heating of the sample due to photoexcitation can be substantial. Since dielectric substrates have superior thermal conductivity to these low dimensional systems, and the continuous heating is governed by the sample's thermal conductivity, high quality thin films would be advantageous. This is one of the motivations for selecting large area thin films of $\mathrm{K}_{0.3} \mathrm{MoO}_{3}$.

The second, equally important motivation for growing thin films is the rapid development of time-resolved structural probes, in particular, femtosecond electron diffraction (FED), where for the experiments in the transmission mode free standing crystalline films are required. This technique, which has been first used to follow the temporal evolution of laser induced melting in thin metal films on a ps time-scale, ${ }^{11}$ harbors great promise for resolving the structural dynamics with atomic level detail. While one approach to obtain free standing thin films is to use cleaving techniques ${ }^{12}$ of single crystals, the second approach would be to deposit thin films on soluble substrates, as in the case of Bi films used in FED experiments, ${ }^{13}$ which were prepared on $\mathrm{NaCl}$ crystals.

Van der Zant et al. have successfully applied the pulsed laser deposition (PLD) technique ${ }^{14-18}$ to grow films of CDW system $\mathrm{Rb}_{0.3} \mathrm{MoO}_{3}$ with thickness of the order of $1 \mu \mathrm{m}$. However, for the time-resolved $\mathrm{THz}$ conductivity measurements, as well as for FED experiments, the films should be about one order of magnitude thinner. In the following, we present the results of pulsed laser deposition, characterization and fsTRS measurements on thin films of $\mathrm{K}_{0.3} \mathrm{MoO}_{3}$ with thickness of the order of $100 \mathrm{~nm}$. This paper is organized as follows: In Sec. II we describe the method and parameters of the thin film deposition. In Sec, III we present the results of film characterization by UV-vis transmission, grazing incidence X-ray diffraction (XRD), atomic force microscopy (AFM), and electric transport measurements, while in Sec. IV the results of fsTRS on selected films are presented and compared with the ones for single crystals. Finally, in Sec. V, some implications of the above results to the physics of CDWs are pointed out.

\section{THIN FILMS GROWTH}

PLD technique is successfully applied to grow thin films of several types of materials, including semiconductors, fer- roelectrics, and high- $T_{\mathrm{C}}$ superconductors. ${ }^{19}$ The schematic of PLD setup is presented in Fig. 1.

The targets were prepared by grinding $\mathrm{K}_{0.3} \mathrm{MoO}_{3}$ single crystalline samples and pressing into pellets. Both targets and substrates were placed into a vacuum chamber that was first evacuated down to a residual pressure of $10^{-4} \mathrm{~Pa}$. Then, a flux of oxygen was circulated with a dynamic pressure strictly monitored with a MKS50 flow controller. A pulsed $\mathrm{KrF}^{*}$ excimer laser source (COMPexPro) was used to generate plasma plume by ablation from a rotating polycrystalline target. The substance in plasma plume was deposited on the substrate that was mounted on a heater block placed parallel to the target at a $5 \mathrm{~cm}$ separation distance. The stoichiometry and morphology of the deposited films strongly depends on the thermodynamic conditions during the film growth. The most important parameters include the substrate temperature, the oxygen pressure in the chamber, and the deposition rate.

Based upon previous experience ${ }^{14-16}$ with the structurally very similar compound $\mathrm{Rb}_{0.3} \mathrm{MoO}_{3}$, oxygen pressure $\left(p_{\mathrm{O} 2}\right)$ has been varied between 1 and $10 \mathrm{~Pa}$ and the substrate temperature $\left(T_{\mathrm{S}}\right)$ between $400^{\circ} \mathrm{C}$ and $450{ }^{\circ} \mathrm{C}$ with several attempts at $350{ }^{\circ} \mathrm{C}$ and $500^{\circ} \mathrm{C}$. Depositions for each set of conditions were performed on both (510) $\mathrm{SrTiO}_{3}$ (STO) and (1-102) $\mathrm{Al}_{2} \mathrm{O}_{3}$ (ALO) substrates, which have suitable lattice parameters. ${ }^{14}$ The laser pulse parameters have been kept constant at $248 \mathrm{~nm}$ wavelength and $25 \mathrm{~ns}$ pulse width, with repetition rate of $5 \mathrm{~Hz}$ and pulse energy of $250 \mathrm{~mJ}$ corresponding to a fluence of $2.4 \mathrm{~J} / \mathrm{cm}^{2}$. The number of pulses was 6000 for a nominal $100 \mathrm{~nm}$ thickness of films, except for two series with 15000 pulses. Altogether, nine series of depositions on twin STO and ALO substrates have been carried out.

\section{RESULTS OF STANDARD CHARACTERIZATION METHODS}

In the following, we describe the results of several characterization methods used to determine sample quality, including optical properties, crystal structure, stoichiometry, morphology, and transport properties in order to select the most appropriate ones for fsTRS measurements.

$\mathrm{UV}$-vis transmission and grazing incidence XRD have been measured on all prepared films. Only two series of films have demonstrated a good agreement with bulk XRD (structural) and optical spectra of $\mathrm{K}_{0.3} \mathrm{MoO}_{3}$ on both STO and

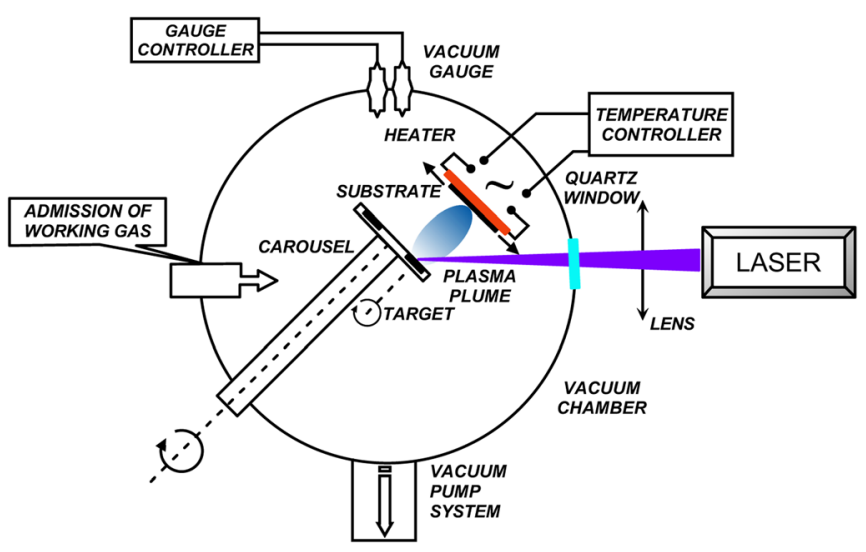

FIG. 1. (Color online) Schematic of PLD setup. 
ALO substrates. For both series $p_{\mathrm{O} 2}$ has been set to $4 \mathrm{~Pa}$, while $T_{\mathrm{S}}$ has been set to $400{ }^{\circ} \mathrm{C}$ and $450{ }^{\circ} \mathrm{C}$ and the number of laser pulses hitting the target has been set to 6000 and 15000 , respectively. Those two series will be referenced as BB11 and BB14, respectively. In the following, we will present the results of different characterization techniques on these four films.

\section{A. Time-of-flight elastic recoil detection analysis- stoichiometry}

Time-of-flight elastic recoil detection analysis (TOFERDA) was used to determine average film thickness and stoichiometric relations between potassium $(\mathrm{K})$, molybdenum (Mo), and oxygen (O) atoms in the deposited film. In TOFERDA energy of ions recoiled from the sample by incident, heavy ion beam is detected in coincidence with time of flight of each recoil between two time detectors separated at 523 mm. $\mathrm{K}_{0.3} \mathrm{MoO}_{3}$ film was bombarded with $20 \mathrm{MeV}^{127} \mathrm{I}^{6+}$ beam. The angle between the beam and the sample was $20^{\circ}$. The scattered and recoiled ions were detected under $37.5^{\circ}$. Different masses are separated (mass separation is 1 up to $M=28$ ) as can be seen from Fig. 2, where each branch represents different mass (sample constituent). Further analysis reveals distribution of each element atoms through the film depth, part of the substrate close to the surface, as well as possible contamination of the film.

Atomic concentrations of $\mathrm{K}, \mathrm{Mo}$, and $\mathrm{O}$ atoms were determined together with the film thickness, the latter being $110 \pm 10 \mathrm{~nm}$ for two BB11 films and $320 \mathrm{~nm}$ for BB14 film on ALO. The atomic ratio of $\mathrm{K}$ and Mo was $0.26: 1$ and $0.25: 1$, while the atomic ratio of $\mathrm{O}$ and Mo was 3.7:1 and $4: 1$, respectively. Traces of hydrogen and carbon are present on the film surface, while the substrate atoms, either aluminum (Al) or strontium ( $\mathrm{Sr}$ ) and titanium (Ti) are present only in the substrate, i.e., beneath the $\mathrm{K}_{0.3} \mathrm{MoO}_{3}$ film.

A lower content of $\mathrm{K}$ atoms can be related to the presence of insulating $\mathrm{MoO}_{3}$ in the films. On the other hand, increased oxygen content cannot be explained by the formation of molybdenum oxides or by the hydration of $\mathrm{MoO}_{3}$, but it has also been observed in $\mathrm{Rb}_{0.3} \mathrm{MoO}_{3}$ for higher oxygen ambient pressures. ${ }^{15}$

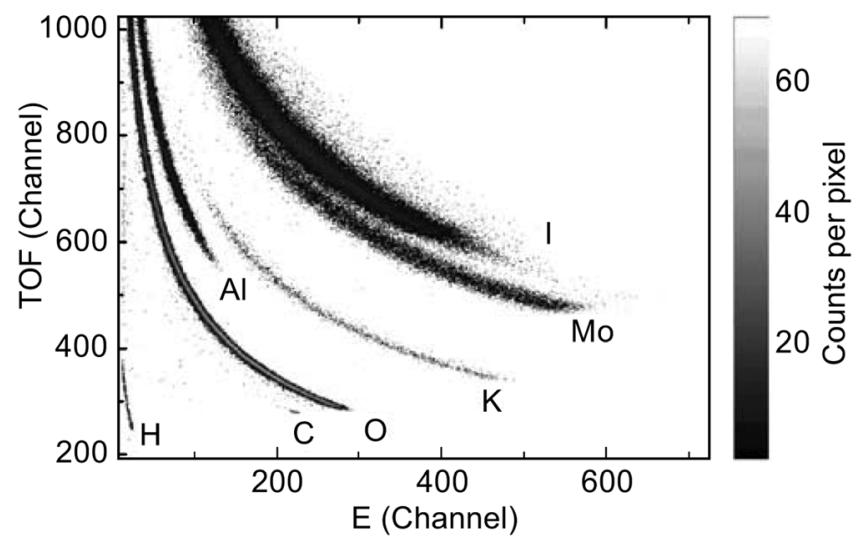

FIG. 2. TOF-ERDA coincidence map obtained by bombarding BB14 film on ALO substrate with $20 \mathrm{MeV}^{127} \mathrm{I}^{6+}$ beam. Each trace in the map corresponds to the different sample element as noted.

\section{B. Atomic force microscopy}

AFM imaging was performed using a Multimode AFM with Nanoscope IIIa controller (Bruker, Billerica, USA) with a vertical engagement (JV) $125 \mu \mathrm{m}$ scanner. Contact mode imaging was performed using silicon-nitride tips (NP-20, Bruker, nom. freq. $56 \mathrm{KHz}$, nom. spring constant of $0.32 \mathrm{~N} / \mathrm{m}$ and ORC8-10, Bruker, nom. freq. $50-90 \mathrm{kHz}$, nom. spring constant of $0.73 \mathrm{~N} / \mathrm{m}$ ). The linear scanning rate was optimized between 1.5 and $2 \mathrm{~Hz}$ with scan resolution of 512 samples per line. Processing and analysis of images were carried out using NanoScope ${ }^{\mathrm{TM}}$ software (Bruker, Billerica, USA). All images presented are raw data except for first order two-dimensional flattening. Measurements were performed in the air at ambient temperature and humidity.

Characteristic topography of $\mathrm{K}_{0.3} \mathrm{MoO}_{3}$ films on STO and ALO substrates is presented as height data in Fig. 3. These images are representative for both BB11 and BB14 films.

$\mathrm{K}_{0.3} \mathrm{MoO}_{3}$ film on STO substrate consists of a collection of amorphous (oblong shape) nanoparticles (grains) with no preferential orientation. Grain size varies between 100 and $250 \mathrm{~nm}$. Estimated grain height is in the range of 2-6 nm with several larger grains (up to $15 \mathrm{~nm}$ ). The surface roughness is $2.2 \mathrm{~nm}$ rms.

$\mathrm{K}_{0.3} \mathrm{MoO}_{3}$ film on ALO substrate consists of a collection of crystallites (rectangular shaped grains with sharp edges) with in plane dimensions of $200-400 \mathrm{~nm}$ and 6-18 nm height. The surface roughness is $7.2 \mathrm{~nm}$ rms.

\section{Grazing incidence X-ray diffraction}

Grazing incidence X-ray diffraction (GIXRD) data were measured using a set-up with a sealed tube ( $\mathrm{Cu}$ anode) as a source, and a homemade detection camera with fine adjustment of grazing incidence angle. ${ }^{20}$ The GIXRD curve(s) were acquired with a position sensitive detector (Hecus PSD-50M). Fixed incidence angle of $\mathrm{Cu}-\mathrm{K}_{\alpha}$ radiation $(\lambda=1.54 \AA)$ with respect to the sample surface was $\alpha_{\mathrm{I}}=1.0^{\circ}$, which was large enough to allow the X-rays to penetrate throughout the whole film and small enough to reduce the substrate contribution.

Figure 4 shows the GIXRD data obtained from BB14 film on ALO substrate, compared to the crystallographic data for monoclinic $\mathrm{K}_{0.3} \mathrm{MoO}_{3}$ powder phase, ${ }^{21}$ which is characteristic for all films grown both on ALO and STO

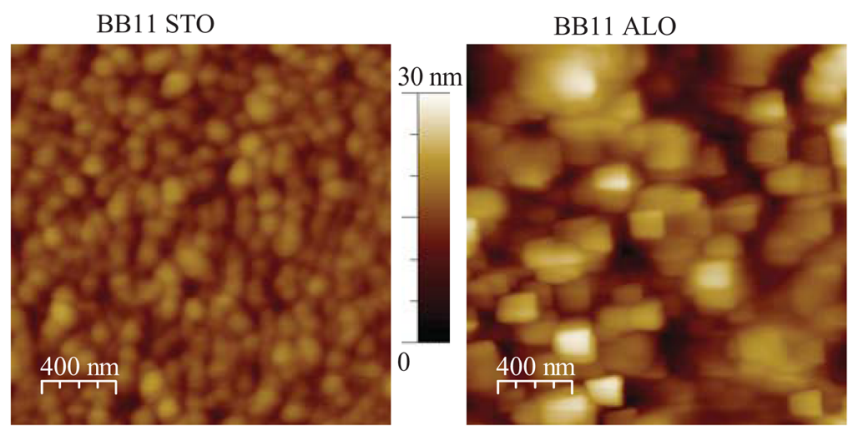

FIG. 3. (Color online) Topography of $\mathrm{K}_{0.3} \mathrm{MoO}_{3}$ films on STO (left panel) and ALO (right panel) substrates obtained by AFM imaging. Scan sizes are $2 \mu \mathrm{m} \times 2 \mu \mathrm{m}$ with vertical scales of $30 \mathrm{~nm}$. 


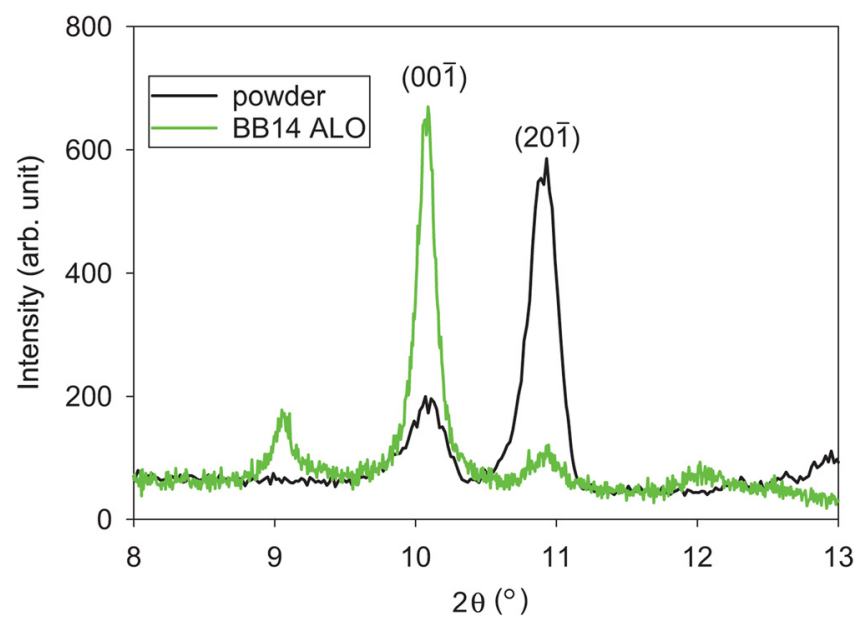

FIG. 4. (Color online) GIXRD spectrum of a $\mathrm{K}_{0.3} \mathrm{MoO}_{3}$ film grown on ALO substrate (dots), together with the calculated XRD spectrum for target powder $\mathrm{K}_{0.3} \mathrm{MoO}_{3}$ (red line).

substrates. Two peaks in the GIXRD pattern, marked as 001 and 20-1 in Fig. 4 correspond to the Bragg peaks of the monoclinic $\mathrm{K}_{0.3} \mathrm{MoO}_{3}$ phase. Intensity ratio of these reflections suggests that $\mathrm{K}_{0.3} \mathrm{MoO}_{3}$ grains are textured with (001) planes preferably orientated parallel with the substrate surface. This means that the most conducting (chain) direction and the least conducting direction lay in the plane parallel with the substrate. Results show that the same conclusion holds for other investigated films. One has to bear in mind, though, that the evidenced (001) texture is only indicative, as the experiments have been performed for only one angle of incidence.

\section{Transport measurements: Electrical resistivity}

Resistivity measurements have been performed on bulk sample and on several films grown on both substrates in the temperature range $100 \mathrm{~K}-300 \mathrm{~K}$ (Fig. 5). Bulk sample has been measured in the standard four contacts configuration, while thin films have been measured in two contact, mechanically patterned four contacts and van der Pauw configuration in several areas of the films. Electrical contacts have been made with thin copper wires attached with silver paint on evaporated gold pads. The lengths and widths of the sample area were of the order of $1 \mathrm{~mm}$ and $0.5 \mathrm{~mm}$, respectively.

The resistivity $(\rho)$ of $\mathrm{K}_{0.3} \mathrm{MoO}_{3}$ is highly anisotropic, with $\rho \sim 5 \times 10^{-6}-5 \times 10^{-5} \Omega \mathrm{m}$ in the most conducting direction ${ }^{22}$ and the anisotropy ratio ${ }^{23}$ of about 1:30:1000. The measured resistance of $100 \mathrm{~nm}$ thick BB11 films was of the order of G $\Omega$ corresponding to $\rho \sim 100 \Omega \mathrm{m}$, while the resistance of $320 \mathrm{~nm}$ thick BB14 films was of the order of $100 \mathrm{k} \Omega-1 \mathrm{M} \Omega$ corresponding to $\rho \sim 0.03-0.3 \Omega \mathrm{m}$. In BB11 films $\rho$ increased only slightly (up to two times) from room temperature down to $100 \mathrm{~K}$; while in BB14 films, the temperature variation was more pronounced. We present the results for the BB14 film on ALO with the strongest temperature variation $\rho(T)$ measured in patterned four contacts configuration.

In Fig. 5 the resistivity of both bulk sample and selected film relative to the room temperature value is presented in the inset, while the main panel shows the derivative of $\rho$ in respect to inverse temperature, $d \ln \left(\rho / \rho_{R T}\right) / d(1 / T)$. In semiconductors, the temperature dependence of resistivity is thermally activated and the value of the activation energy $\Delta$, or half of the gap value, corresponds to constant value of the derivative.

Therefore, the constant value of the derivative in a bulk sample at low temperatures demonstrates the semiconducting nature of the $\mathrm{CDW}$ state with $\Delta=450 \mathrm{~K}$. As the temperature increases, the derivative shows a narrow peak indicating the Peierls transition to $\mathrm{CDW}$ state at $T_{\mathrm{C}}=180 \mathrm{~K}$, and becomes negative in metallic state above $T_{\mathrm{C}}$.

None of these three features was present in any of the investigated thin films. In BB14 film presented in Fig. 5 almost linear $\Delta(T)$ dependence, with a slight change of slope around $150 \mathrm{~K}$, was observed. Similar results are obtained by Wang et al. ${ }^{38}$ on sintered polycrystalline samples. Moreover, $\rho(T)$ could be well described by the one dimensional variable range hopping (1D VRH) $\rho \sim \exp \left(-\left(T_{0} / T\right)^{1 / 2}\right)$, as shown in Fig. 6, with fitting parameter $T_{0}=5100 \mathrm{~K}$.

The decrease of $T_{\mathrm{C}}$ along with the smearing of sharp features in $\Delta(T)$ at $T_{\mathrm{C}}$ is characteristic for strongly doped ${ }^{24-26}$ or irradiated ${ }^{27}$ samples of $\mathrm{K}_{0.3} \mathrm{MoO}_{3}$. For instance, the samples doped with about $0.3 \%$ of tungsten (W) (Ref. 26) show a crossover at about $150 \mathrm{~K}$. Moreover, $\rho(T)$ dependence in these samples is more readily described by VRH. ${ }^{24}$ The presence of defects limits the phase coherence volume $V_{\text {coh }}$ and prevents the establishment of long range CDW order. The phase coherence length $l_{\phi}$ along the chain is particularly sensitive to defects and is in nominally pure samples of the order of micrometers. The ratio of coherence lengths is close to $8: 3: 1$, with only slight variations (see Table II in Ref. 28) even upon introducing defects. The coherence volume for nominally pure bulk sample is $\mathrm{V}_{\mathrm{coh}} \sim 10^{8} \mathrm{~nm}^{3}$. However, in the case of granular films, the $l_{\phi}$ limit is given by the grain size. AFM images show that the largest grains have surface dimensions of 100x250 nm. Taking film depth as a maximum grain depth, the uppermost average grain volume is about $\mathrm{V}_{\mathrm{g}} \sim 10^{7} \mathrm{~nm}^{3}$, which is at least an order of magnitude smaller than the phase coherence volume in the pure bulk samples. Moreover, by

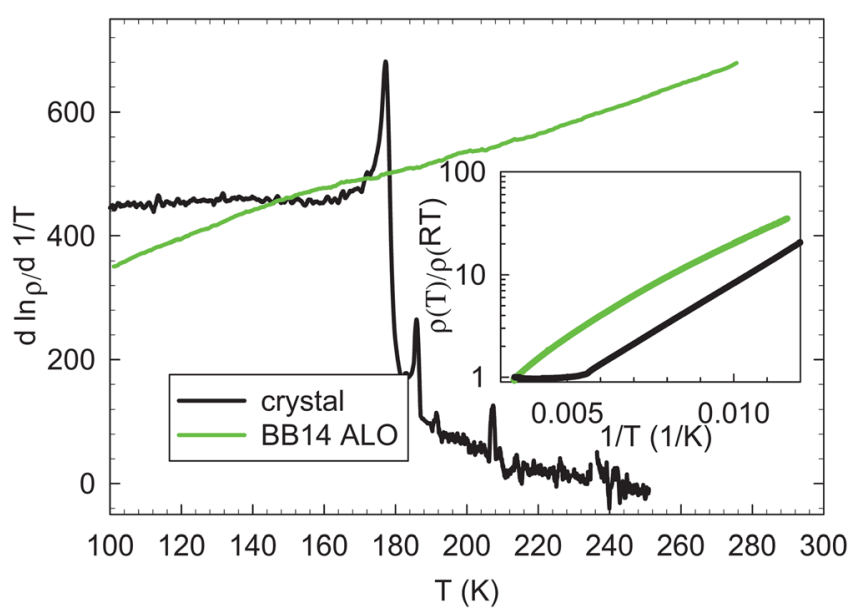

FIG. 5. (Color online) Electric transport measurements on $\mathrm{K}_{0.3} \mathrm{MoO}_{3}$ bulk and on BB14 film on ALO substrate. In the inset is the resistivity relative to the room temperature value, while in the main panel is the derivative of the logarithm of resistivity in respect to inverse temperature. 


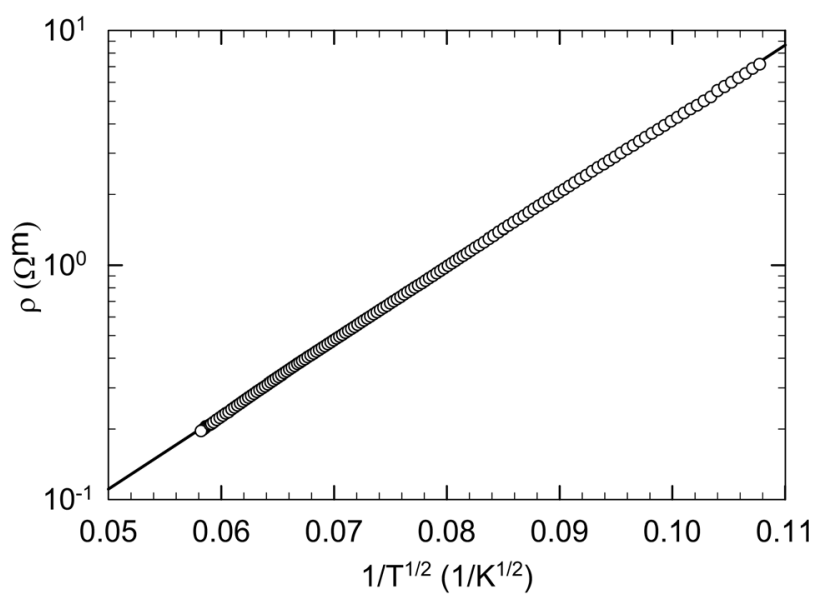

FIG. 6. Resistivity of BB14 film on ALO substrate presented as the function of $T^{-1 / 2}$ indicating 1D VRH behavior (circles). Solid line is a fit to 1D VRH behavior.

assuming that the approximate 8:3:1 scaling between the correlation lengths in different directions ${ }^{28}$ is valid also in granular thin films, a rough estimate gives $V_{\text {coh }} \sim 3 \times 10^{4}-6 \times 10^{5} \mathrm{~nm}^{3}$, which is between the values obtained in High Resolution XRD measurements of heavily doped samples ${ }^{28}$ and lightly irradiated ones. $^{29}$ The smearing of the transition, the reduction of $T_{\mathrm{C}}$, and the VRH temperature dependence have also been observed in nanometer sized samples of CDW systems o- $\mathrm{TaS}_{3}$ and $\mathrm{NbSe}_{3} .{ }^{30}$

High resistivity of BB14 films is of the order of magnitude expected for the least conducting direction, which for the observed grain growth morphology lies in the measurement plane and therefore contributes dominantly to the resistivity. In addition, reduced $V_{\text {coh }}$ can also contribute to the increase in resistivity, as observed in doped samples. ${ }^{25}$ However, for thinner BB11 films, the resistivity is 2-3 orders of magnitude higher and probably dominated by disjoint grains and/or grain boundaries.

\section{RESULTS OF FEMTOSECOND TIME-RESOLVED SPECTROSCOPY}

We studied both the temperature and excitation density dependence of photoinduced (PI) reflectivity changes using an optical pump-probe technique. A Ti:sapphire regenerative amplifier producing $40 \mathrm{fs}$ laser pulses at $\lambda=800 \mathrm{~nm}$ (photon energy of $1.55 \mathrm{eV}$ ) at a $250 \mathrm{kHz}$ repetition rate was used as a source of both pump and probe pulse trains. The pump and probe laser beams were polarized orthogonally to reduce the noise due to pump scattered light. The induced changes in reflectivity $(R)$ were recorded utilizing a fast-scan technique, enabling high signal-to-noise levels. ${ }^{31}$ The experiments were performed on four selected thin films of $\mathrm{K}_{0.3} \mathrm{MoO}_{3}(\mathrm{BB} 11$ and BB14 both on STO and ALO substrates) for which the standard characterization methods suggested the films to be granular $\mathrm{K}_{0.3} \mathrm{MoO}_{3}$.

We have scanned through the films and searched for the characteristic PI reflectivity trace of $\mathrm{K}_{0.3} \mathrm{MoO}_{3}$ - in particular, the benchmark was the presence of the oscillatory signal due to coherently excited phonon modes, characteristic for $\mathrm{K}_{0.3} \mathrm{MoO}_{3} \cdot{ }^{32}$ For both BB14 films, the PI signal showed strong variation along the film, with characteristic $\mathrm{K}_{0.3} \mathrm{MoO}_{3}$ signal showing up only in the area about $2 \mathrm{~mm}$ from the edge of the film. On the other hand, BB11 films were much more homogeneous, showing the characteristic $\mathrm{K}_{0.3} \mathrm{MoO}_{3}$ response throughout the $10 \times 10 \mathrm{~mm}^{2}$ film (the transients were identical to the ones obtained at the edge of the BB14 films). Following the scanning of the sample area, we have performed detailed temperature and excitation density dependence studies of the optical response.

The pump and probe beams were focused onto the sample in spots of $d_{\text {pump }}=(90 \pm 4) \mu \mathrm{m}$ and $d_{\text {probe }}=(45 \pm 2) \mu \mathrm{m}$ diameter to ensure a homogeneous excitation profile. The samples were mounted in an optical cryostat and the temperature was varied between 4 and $300 \mathrm{~K}$. Probe polarization dependent measurements on all films showed that the PI signal was isotropic with respect to the film direction, in strong contrast to single crystals where pronounced anisotropy is observed. $^{32}$

\section{A. Temperature dependence of the photoinduced reflectivity transients}

We plot in Fig. 7 the temperature dependence of the PI reflectivity change as a function of temperature and time after photoexcitation. The recorded $\Delta R / R$ traces on thin films are qualitatively similar to those recorded on single crystals. We note however that (a) the amplitude of the induced change was substantially lower than in the crystals, despite the fact that films are optically thick (in optically thin films the reflectivity and its photoinduced change depend on the film thickness and on the refractive index of the substrate via Fresnel equations, therefore straightforward comparison of $\Delta R / R$ would be unjustified), and (b) the damping of the coherently excited phonon modes is also substantially stronger than in the case of single crystals. Similar results have been observed for all four films.

The PI reflectivity changes in films are about a factor of 50 smaller than in the case of single crystals at the same excitation density. We suggest that this could be attributed to
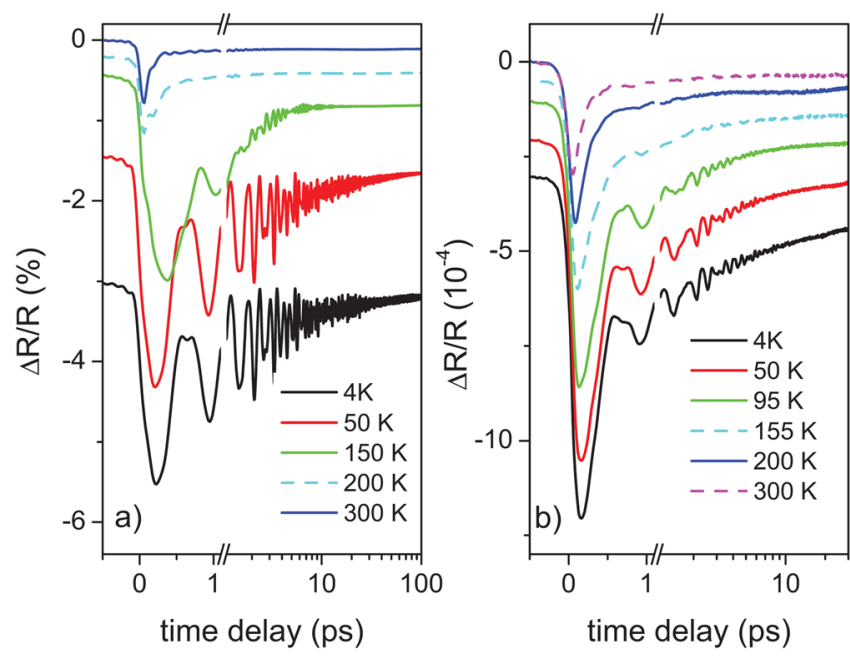

FIG. 7. (Color online) The PI reflectivity transients in (a) bulk $\mathrm{K}_{0.3} \mathrm{MoO}_{3}$ and (b) in BB14 film on ALO substrate as a function of temperature recorded at the excitation density of $20 \mu \mathrm{J} / \mathrm{cm}^{2}$ as a function of temperature. 
the different crystal orientation of $\mathrm{K}_{0.3} \mathrm{MoO}_{3}$ grains in thin films in respect to single crystals. The experiments on single crystals were performed on freshly cleaved $\mathrm{K}_{0.3} \mathrm{MoO}_{3}$ single crystals with the laser polarization in the (20-1) plane, i.e., perpendicular to the least conducting direction. ${ }^{1}$ On the other hand, GIXRD measurements on thin films suggest that $\mathrm{K}_{0.3} \mathrm{MoO}_{3}$ grains are textured such that (001) planes are mostly oriented parallel to the surface plane. Given the strong crystalline anisotropy, which results also in a pronounced anisotropy of the PI reflectivity change within the (20-1) plane, ${ }^{32}$ it is very likely that the difference in the observed magnitudes of the PI changes in single crystals and thin films is due to different orientations of the two. Unfortunately, neither the equilibrium optical data nor time-resolved reflectivity data on single crystals with polarization perpendicular to the (20-1) plane are available.

The non-oscillatory part of the data was found to fit well to a sum of three exponential functions with well separated decay times in the whole temperature range:

$$
S(t)=A_{1}(t) e^{-t / \tau_{1}}+A_{2}(t) e^{-t / \tau_{2}}+A_{3}(t) e^{-t / \tau_{3}} .
$$

The component $A_{3}$, which is fitted (approximated) with an exponential decay with a time constant $\tau_{3}$ of several $100 \mathrm{ps,}$ corresponds to a diffusive thermal recovery of the system (bolometric response) and will not be discussed in detail. During fitting, $\tau_{3}$ was fixed to a constant temperature and excitation independent value of $\tau_{3}=400$ ps. The fastest component, $A_{1}$, whose relaxation time $\tau_{1}$ is on the $100 \mathrm{fs}$ time scale, shows in single crystals critical slowing down upon approaching the critical temperature from below. It has been recently identified as an over-damped mode of the electronic part of the order parameter, ${ }^{3}$ describing the recovery of the perturbed conduction electron modulation on the time scale comparable to the characteristic time of the lattice motion (phonon period). The component $A_{2}$, whose relaxation time, $\tau_{2}$, is found to decrease as the critical temperature is
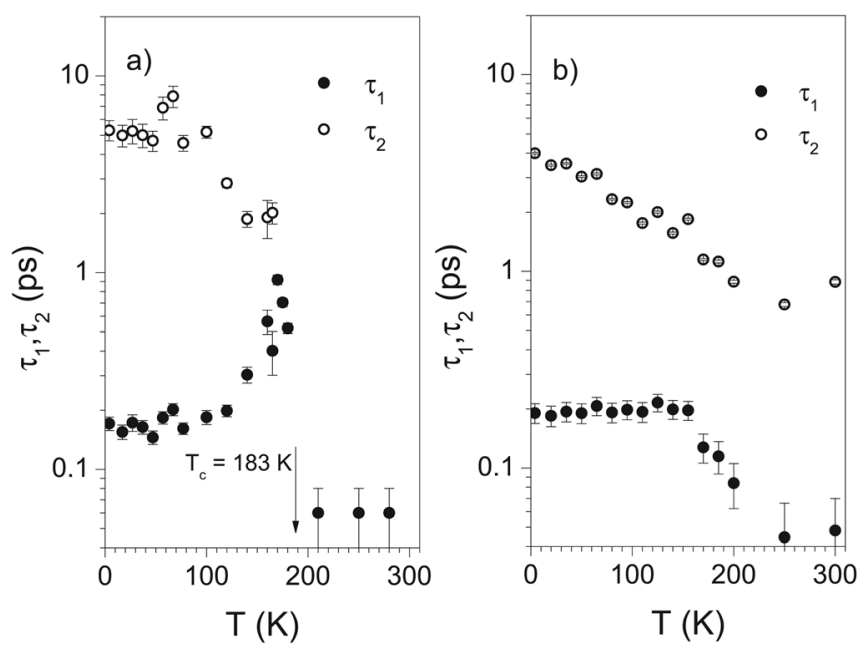

FIG. 8. Comparison of the temperature dependences of the two characteristic timescales, $\tau_{1}$ and $\tau_{2}$, obtained on single crystal (a) and BB14 film on ALO (b). In thin film no characteristic slowing down of $\tau_{1}$ is observed. Moreover, as opposed to single crystals, no sharp change of the characteristic timescales near $T_{\mathrm{C}}$ is noticed.

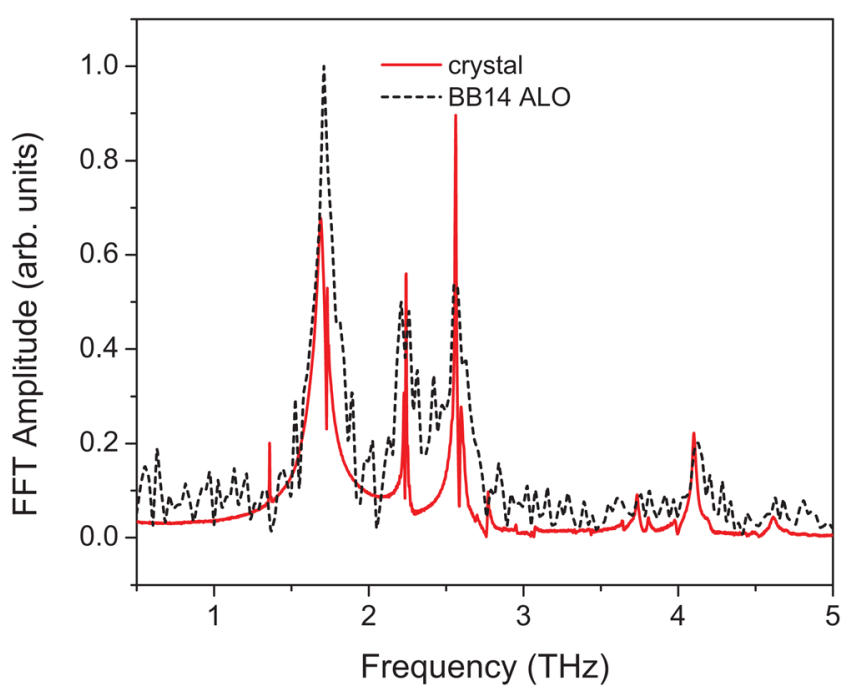

FIG. 9. (Color online) Comparison between the FFT spectra measured in a $\mathrm{K}_{0.3} \mathrm{MoO}_{3}$ crystal and film on $\mathrm{Al}_{2} \mathrm{O}_{3}$ substrate recorded at $10 \mathrm{~K}$.

approached from below, is attributed to the recovery of the coupled electron-lattice CDW order parameter. ${ }^{3}$

Figure 8 shows the comparison of the temperature dependencies of the two characteristic relaxation times for (a) single crystal and (b) BB14 film on ALO. The relaxation time $\tau_{2}$ has similar value and temperature dependence as in the crystal, decreasing with increasing temperature. The fast relaxation time $\tau_{1}$ in thin films has a similar value as in the crystal at high and low temperature. However, where in single crystals $\tau_{1}$ shows a divergence near $T_{\mathrm{C}}=180 \mathrm{~K}$, in thin films it shows almost no temperature dependence up to 150 $\mathrm{K}$, when starts to decrease steadily. The absence of the pronounced anomaly suggests that the grains have different values of $T_{\mathrm{C}}$.

A very similar quenching of $\tau_{1}$ divergence has also been observed as a result of the topology change in $\mathrm{NbSe}_{3},{ }^{33}$ which is a CDW material that crystallizes in whisker and ring crystals. Shimatake et al. offered an explanation of the $\tau_{1}$ quenching in small ring crystals in terms of enhanced phase fluctuations in the closed-loop topology. ${ }^{33}$ Obviously, this explanation cannot be extended to the case of $\mathrm{K}_{0.3} \mathrm{MoO}_{3}$ films; however, it does raise questions about how finite sizes of CDW crystals affect the Quasy Particle dynamics.

Fast Fourier transform (FFT) analysis on the reflectivity transients was performed in order to evaluate the temperature dependence of the oscillatory signal. The FFT spectra obtained at $10 \mathrm{~K}$ on both single crystal and thin film are plotted in Fig. 9 for comparison. There is an overall good agreement between the low temperature spectra on film and crystal, as the four strongest modes are observed in both. On the other hand, the modes in the film are highly asymmetric and their damping (linewidth) is much stronger. We attribute this to the finite grain size and inhomogeneity of the films, acting like impurities in a crystal.

The temperature dependence of the FFT spectra is plotted in Fig. 10, where one can see that the temperature dependence of spectra measured on films and crystal are also very similar. 


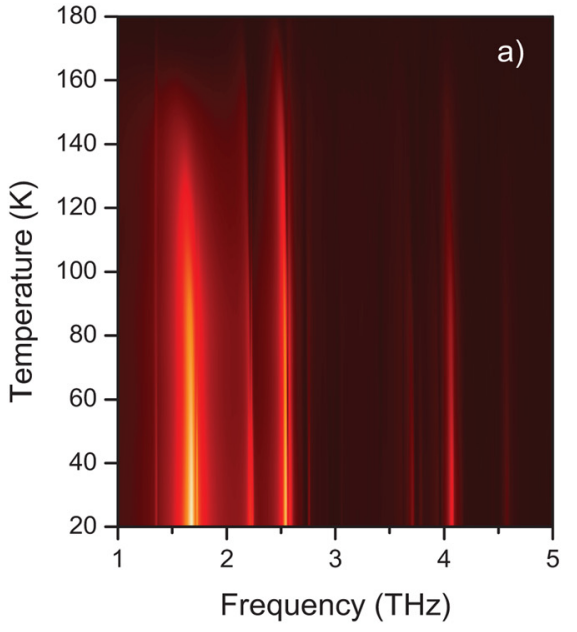

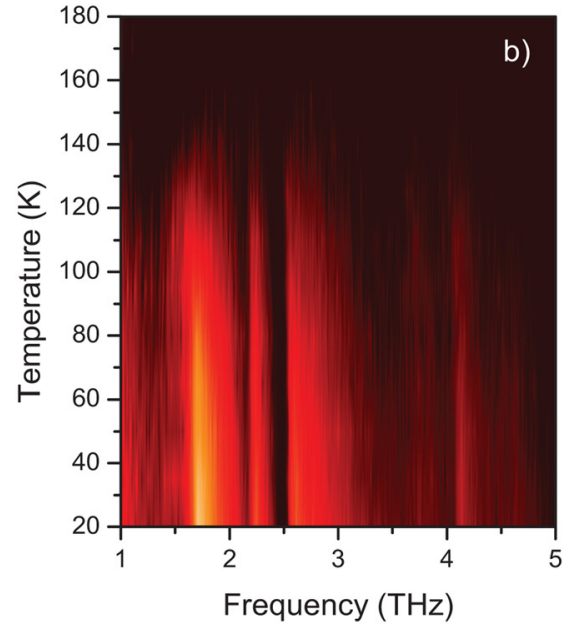

FIG. 10. (Color online) The temperature dependence of the FFT spectra of the PI signal in (a) $\mathrm{K}_{0.3} \mathrm{MoO}_{3}$ single crystal and (b) BB14 film on ALO substrate. The intensity map for both is on the linear scale.
One noteworthy difference is, however, that the low temperature modes in $\mathrm{K}_{0.3} \mathrm{MoO}_{3}$ film vanish at considerably lower temperatures than in the case of the single crystal. This observation, combined with the temperature dependence of the incoherent response (Fig. 8), suggests that the critical temperature in thin films is lower than that in the single crystal. Due to the absence of sharp anomalies in the incoherent response in the thin film, it is difficult to determine precisely the critical temperature. It seems, however, to be about $30 \mathrm{~K}$ lower than in the single crystal.

\section{B. Excitation density dependence of the photoinduced reflectivity transients at $10 \mathrm{~K}$}

Following the elaborate study ${ }^{2}$ of the dependence of PI reflectivity on photoexcitation density in single crystals of $\mathrm{K}_{0.3} \mathrm{MoO}_{3}$, we have performed a similar study also on thin films. It has been shown that the photoinduced melting of the electronic part of the order parameter can be determined from such studies. The threshold energy could be estimated from the excitation density dependence of the maximum photoinduced reflectivity change, which showed saturation above the threshold excitation density. The maximum PI reflectivity change of $\sim 18 \%$ equals the reflectivity difference between the low temperature CDW state and normal state $\left(\mathrm{T}>\mathrm{T}_{\mathrm{C}}\right)$ and implies that at fluences above this threshold fluence, electronic modulation is transiently collapsed. In the normal state the PI reflectivity signal is considerably smaller (see Fig. 7) and shows simple linear dependence on the excitation fluence. Similarly, the fast relaxation time scale $\left(\tau_{1}\right)$ showed critical slowing down upon increasing the excitation fluence, followed by an abrupt drop, once the threshold fluence was reached. ${ }^{2}$ In $\mathrm{K}_{0.3} \mathrm{MoO}_{3}$ single crystals, this energy was found to be comparable to the electronic part of the condensation energy, as calculated assuming the mean field theory. ${ }^{1}$ Figure 11 presents the comparative data of the excitation density dependence of the reflectivity transients recorded in (a) single crystal and (b) BB14 film on ALO substrate.

While qualitative behavior of the two is very similar, there are clear differences in the two data sets that need to be discussed. First of all, as mentioned above, the PI reflectivity change in films is about a factor of 50 smaller than in crystals. Secondly, while clear saturation of the PI reflectivity change at about $18 \%$ is observed in single crystals, no clear saturation is observed in films. Detailed analysis of the amplitude and relaxation time $\left(\tau_{1}\right)$, however, shows the presence of the characteristic excitation density also in the case of thin films.

Figure 12 presents the excitation intensity dependence of the reflectivity transient amplitude and the fast relaxation time $\left(\tau_{1}\right)$ for single crystals and thin films. As discussed above, in single crystals both clear saturation of the amplitude of the PI transient, shown in Fig. 12(a), as well as relaxation time anomaly, shown in Fig. 12(c), demonstrate the PI melting of the electronic part of the order parameter at a characteristic excitation density of $\sim 0.17 \mathrm{~mJ} / \mathrm{cm}^{2}$. In thin films, changes in the relaxation time scale are also evident at comparable characteristic excitation fluence - see Fig. 12(d)_yet no critical slowing down is observed as in the case of a single crystal.
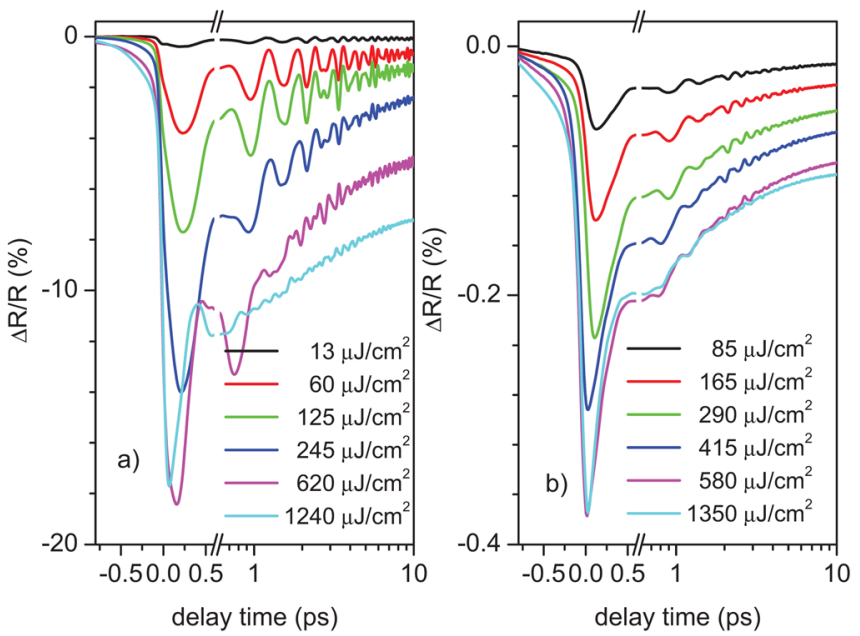

FIG. 11. (Color online) PI changes of reflectivity in $\mathrm{K}_{0.3} \mathrm{MoO}_{3}$ single crystal (a) and BB14 film on ALO substrate (b) as a function of excitation fluence recorded at $10 \mathrm{~K}$. For thin film, the data recorded at $1.35 \mathrm{~mJ} / \mathrm{cm}^{2}$ were divided by a factor of 2 for presentation purpose. 

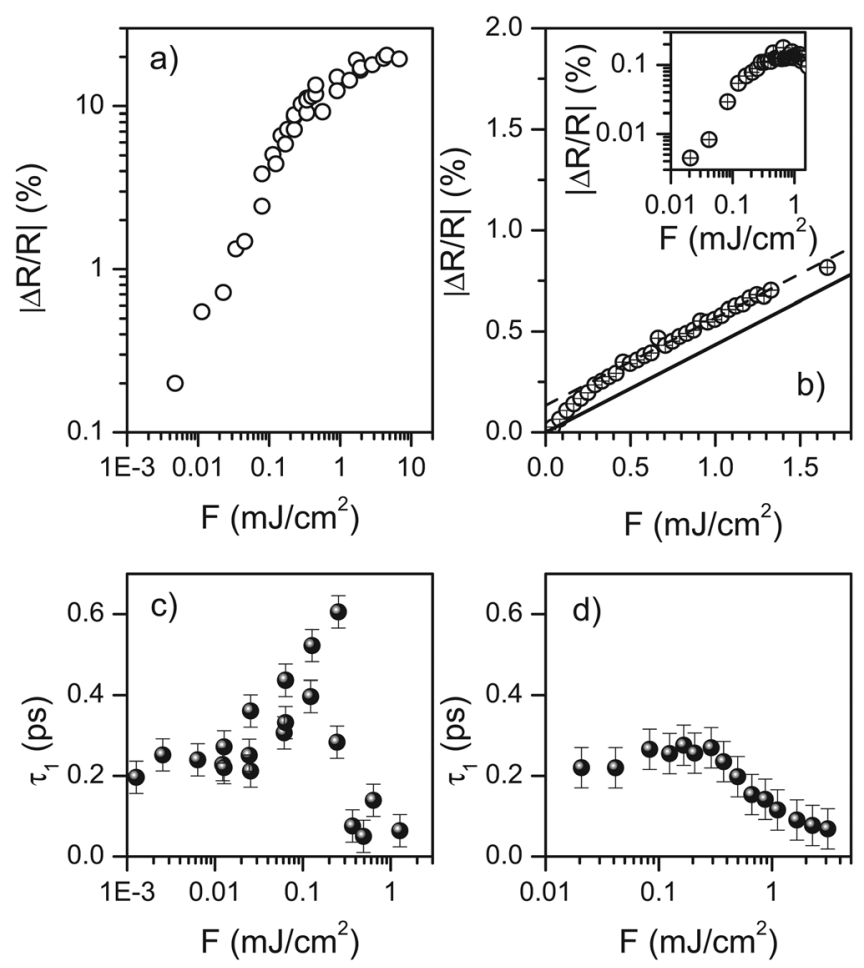

FIG. 12. Comparison of the photoexcitation intensity dependence of the reflectivity transient amplitude and fast relaxation time, $\tau_{1}$, in single crystal [panels (a) and (c), respectively] and BB14 film on ALO substrate [panels (b) and (d), respectively], recorded at $10 \mathrm{~K}$. The fluence dependence of the maximum reflectivity change in thin films reveals the sub-linear dependence at low fluences; while above $0.5 \mathrm{~mJ} / \mathrm{cm}^{2}$, the dependence becomes linear. After subtracting (see the discussion in text) the linear contribution (solid line) from the data, the part showing saturation behavior is revealed [inset to panel (b)], with similar threshold fluence as observed in single crystals.

The fluence dependence of the PI reflectivity amplitude in films, plotted in Fig. 12(b), shows a sub-linear dependence at low fluences, while above $\sim 0.5 \mathrm{~mJ} / \mathrm{cm}^{2}$ the dependence is linear. Such behavior can be attributed to much stronger temperature dependence of the dielectric function in the normal state (above $T_{\mathrm{C}}$ ) in thin films, as opposed to single crystals. To illustrate this effect, we can consider that photoexcitation with a femtosecond optical pulse results simply in the electron temperature increase. This is a major oversimplification, especially for materials with a low energy gap in the electronic density of states, so it should only be used as an illustration. In the normal state reflectivity can be approximated by a linear function of temperature, $R\left(T>T_{\mathrm{C}}\right)=R_{0}+\alpha T$, where $\alpha$ is of the order of $10^{-5} \mathrm{~K}^{-1}$. This contribution we will refer to as the single particle contribution. Upon photoexcitation reflectivity, change is then proportional to an increase in electron temperature (experimentally, in the normal state $\Delta R$ is simply proportional to excitation density over many orders of magnitude). Below $T_{\mathrm{C}} \mathrm{CDW}$ gap opens and contribution proportional to $\Delta^{2}(T)$ appears in the $R(T)$ —see, e.g. Ref. 3 along with the single particle contribution (same as the one present in the normal state). Therefore, the temperature dependence of reflectivity over the entire temperature range is given by $R(T)=R_{0}+\alpha T+\beta \Delta^{2}(T)$. The last term we will refer to as the CDW contribution.

Let us now consider the excitation dependence of the reflectivity change in CDW state: $\Delta R=R\left(T_{e}\right)-R\left(T_{0}\right)$, where $T_{0}$ is the initial temperature and $T_{\mathrm{e}}$ is the electronic temperature after photoexcitation. If $\alpha$ is negligible $\left(\alpha \ll \beta \Delta^{2}(0) / T \mathrm{c}\right), \Delta R$ should show a clear saturation at the excitation density required to collapse the gap. If, however, $\alpha$ is not negligible, $\Delta R$ should show sublinear (but close to linear) excitation dependence below the threshold fluence, followed by a linear dependence at higher excitation densities. Indeed, such behavior is observed in the data on $\mathrm{K}_{0.3} \mathrm{MoO}_{3}$ thin films-see Fig. 12(a). Similar results are obtained also in studies of the photoinduced superconductor-normal state phase transition in cuprate superconductors probed with visible light. ${ }^{34-36}$

In order to extract the characteristic excitation density, which is required for suppression of the electronic part of the order parameter in $\mathrm{K}_{0.3} \mathrm{MoO}_{3}$ thin films, we have subtracted the linear term from the dependence of the maximum reflectivity change on excitation fluence. The remaining part is plotted in the inset of Fig. 12(b), showing a clear saturation at a comparable excitation fluence, as for the case of single crystal.

Consequently, from the excitation density dependent studies on $\mathrm{K}_{0.3} \mathrm{MoO}_{3}$ thin films, the behavior in films is quite similar to the behavior observed in single crystals. Again, we note that the difference between the responses in films and in crystals are most likely related to the different orientations of the two with respect to the direction and polarization of the light pulses, where both the constants $\alpha$ and $\beta$, as well as their ratio, are substantially different for the two cases.

\section{DISCUSSION}

The aim of our study was to detect the CDW ground state in the thin films of $\mathrm{K}_{0.3} \mathrm{MoO}_{3}$, which were prepared by PLD on different substrates for the first time. Due to their polycrystallinity, the standard characterization tools failed to detect the CDW order, and it was only the femtosecond spectroscopy that has unambiguously proven the existence of the CDW ground state. The films have been produced by PLD technique with the deposition conditions close to the optimal range determined previously for an order of magnitude thicker films of isostructural CDW system $\mathrm{Rb}_{0.3} \mathrm{MoO}_{3}$. The difference in the unit cell dimensions between the two systems $^{37}$ is negligible with respect to the mismatch with the substrate lattice. ${ }^{14}$ However, structural (GIXRD) and morphological (AFM) characterizations have shown that the nanocrystalline grains have grown much smaller (100$250 \mathrm{~nm}$ ) than in the case of $\mathrm{Rb}_{0.3} \mathrm{MoO}_{3}$ thick films (up to $100 \mu \mathrm{m}) .{ }^{15}$ Moreover, the (20-1) plane of $\mathrm{K}_{0.3} \mathrm{MoO}_{3}$ spanned by the most conducting, or chain, [010] direction and the intermediate conducting [102] direction ${ }^{37}$ was tilted away from the substrate plane ${ }^{14,15}$ without any preferential inplane direction.

Such growth of the grains has resulted in very high resistivity of the films, as the least conducting direction is randomly oriented within the plane. Another contribution to such high resistivity might be due to poor grain connectivity and phase coherence reduction in small grains. Altogether, this prevented a clear demonstration of CDW ground state 
by the standard electric transport measurements and therefore the presence of $\mathrm{K}_{0.3} \mathrm{MoO}_{3}$ in the films.

In this respect, the use of fsTRS technique as a characterization tool for complex systems has proven essential in the present study. Utilizing fast-scan technique, we were able to quickly record the temperature and photoexcitation density dependence of the reflectivity transients with high dynamic range. Since typical time scale for data acquisition at each temperature or excitation level is only $2-5 \mathrm{~min}$, the entire temperature or excitation density dependent measurements could be performed within 2-3 h.

fsTRS has unambiguously shown the presence of CDW state in these granular films due to its high sensitivity to the collective excitations of the CDW state. Indeed, both low perturbation temperature-dependence study as well as the study of the photoinduced phase transition in $\mathrm{K}_{0.3} \mathrm{MoO}_{3}$ films showed characteristic signatures of the CDW order at low temperatures and, moreover, demonstrated the differences of thin films in respect to the single crystals.

While the characteristic energy, required to drive the photoinduced phase transition from the CDW ground state to the high temperature metallic state, is found to be comparable in both films and single crystals, we note that temperature dependent studies reveal that in granular films, transition temperature seems to be about $30 \mathrm{~K}$ below the one observed in single crystals. This reduction is qualitatively consistent with the reduction of the phase coherence length in small grains, similarly to the doped samples. ${ }^{24,28}$

The absence of a clear divergence of the damping of the electronic part of the order parameter, i.e., of $\tau_{1}$, suggests that the transition is quite broad, which may be the consequence of either a distribution of energy gaps in different grains (consistent with much stronger damping of collective modes) or the smearing of the transition in reduced spatial dimensions. ${ }^{30}$

\section{CONCLUSIONS}

We reported on the preparation and characterization of $\sim 100 \mathrm{~nm}$ thin films of $\mathrm{K}_{0.3} \mathrm{MoO}_{3}$. Films on both (510) $\mathrm{SrTiO}_{3}$ (STO) and (1-102) $\mathrm{Al}_{2} \mathrm{O}_{3}$ (sapphire) substrates were prepared by pulsed laser deposition technique. Series of thin films were deposited in different conditions of substrate temperature, oxygen pressure and number of pulses and characterized by different techniques including UV-vis transmission, grazing incidence XRD, AFM, electric transport measurements, and fsTRS.

While most of the typical characterization tools showed evidence that the films consists of small $100 \mathrm{~nm}$ grains of $\mathrm{K}_{0.3} \mathrm{MoO}_{3}$, the standard methods, like electrical transport, failed to determine the broken symmetry of the CDW state at low temperatures. It is only the femtosecond pump-probe optical spectroscopy that unambiguously showed the presence of CDW state in these granular films, and also pointed to the differences in respect to the single crystals.

In conclusion, this work has established fsTRS technique as the indispensible tool for the detection and characterization of complex ground states in nano-sized systems.

\section{ACKNOWLEDGMENTS}

The work at Konstanz University was supported by the Alexander von Humboldt Foundation via Sofja-Kovalevskaja Award, Zukunftskolleg and Center for Applied Photonics at the University of Konstanz. The work at Institute of Physics in Zagreb was supported by the Ministry of Science, Education and Sports of the Republic of Croatia through project Nos. 098-1191005-2876 (Z.S. and I.B.R.), 0350352827-2842 (D.D., D.S., and K.B.), 035-0352843-2844 (K.S.), and 098-0982934-2744 (G.P. and V.S.). The work of H.S. and M.Đ. was partly supported by the Federal Ministry of Education and Science of Bosnia and Herzegovina. Part of the mobility costs was supported by the project 06.05/I3600-2010 of the Croatian science foundation, the ECONET program of the Ministry of Foreign and European Affairs of France and the bilateral project between Romania and Slovenia.

${ }^{1}$ G. Grüner, Rev. Mod. Phys. 60, 1129 (1988).

${ }^{2}$ A. Tomeljak, H. Schäfer, D. Städter, M. Beyer, K. Biljaković, and J. Demsar, Phys. Rev. Lett. 102, 066404 (2009).

${ }^{3}$ H. Schäfer, V. V. Kabanov, M. Beyer, K. Biljaković, and J. Demsar, Phys. Rev. Lett. 105, 066402 (2010).

${ }^{4}$ L. Degiorgi, B. Alavi, G. Mihály, and G. Grüner, Phys. Rev. B 44, 7808 (1991).

${ }^{5}$ P. W. G. Travaglini, Phys. Rev. B 30, 1971 (1984).

${ }^{6}$ M. Beck, M. Klammer, S. Lang, P. Leiderer, V. V. Kabanov, G. N. Gol'tsman, and J. Demsar, arXiv:1102.5616v1.

${ }^{7}$ R. A. Kaindl, M. A. Carnahan, D. S. Chemla, S. Oh, and J. N. Eckstein, Phys. Rev. B 72, 060510 (2005).

${ }^{8}$ J. Demsar, R. D. Averitt, A. J. Taylor, V. V. Kabanov, W. N. Kang, H. J. Kim, E. M. Choi, and S. I. Lee, Phys. Rev. Lett. 91, 267002 (2003).

${ }^{9}$ L. S. Bilbro, R. Valdés Aguilar, G. Logvenov, O. Pelleg, I. Bozovic, and N. P. Armitage, Nat. Phys. 7, 298 (2011).

${ }^{10}$ Z. Bihar, D. Staresinic, K. Biljakovic, and T. Sambongi, Europhys. Lett. 40, 73 (1997).

${ }^{11}$ B. J. Siwick, J. R. Dwyer, R. E. Jordan, and R. J. D. Miller, Science 302, 1382 (2003).

${ }^{12}$ M. Eichberger, H. Schäfer, M. Krumova, M. Beyer, J. Demsar, H. Berger, G. Moriena, G. Sciaini, and R. J. D. Miller, Nature 468, 799 (2010).

${ }^{13}$ G. Sciaini, M. Harb, S. G. Kruglik, T. Payer, C. T. Hebeisen, F. J. M. Z. Heringdorf, M. Yamaguchi, M. H. V. Hoegen, R. Ernstorfer, and R. J. D. Miller, Nature 458, 56 (2009).

${ }^{14}$ H. S. J. van der Zant, O. C. Mantel, C. Dekker, J. E. Mooij, and C. Traeholt, Appl. Phys. Lett. 68, 3823 (1996).

${ }^{15}$ O. C. Mantel, H. S. J. van der Zant, A. J. Steinfort, C. Dekker, C. Traeholt, and H. W. Zandbergen, Phys. Rev. B 55, 4817 (1997).

${ }^{16}$ A. J. Steinfort, H. S. J. van der Zant, A. B. Smits, O. C. Mantel, P. Scholte, and C. Dekker, Phys. Rev. B 57, 12530 (1998).

${ }^{17}$ O. C. Mantel, C. A. W. Bal, C. Langezaal, C. Dekker, and H. S. J. van der Zant, J. Appl. Phys. 86, 4440 (1999).

${ }^{18}$ O. C. Mantel, C. A. W. Bal, C. Langezaal, C. Dekker, and H. S. J. van der Zant, Phys. Rev. B 60, 5287 (1999).

${ }^{19}$ Pulsed Laser Deposition of Thin Films, edited by D. B. Chrisey and G. K. Hubler (Wiley-Interscience, New York, 1994).

${ }^{20}$ O. Glatter and O. Kratky, Small Angle X-ray Scattering (Academic, New York, 1982).

${ }^{21}$ J. Graham and A. D. Wadsley, Acta Cryst. B 20, 93 (1966).

${ }^{22}$ W. Fogle and J. H. Perlstein, Phys. Rev. B 6, 1402 (1972); J Dumas and C. Schlenker, Solid State Commun. 45, 885 (1983); G. Mihaly, J. Dumas, and A. Janossy, ibid. 60, 785 (1986).

${ }^{23}$ J. P. Pouget, S. Kagoshima, C. Schlenker, and J. Marcus, J. Phys. (Paris) Lett. 44, L113 (1983).

${ }^{24}$ L. F. Schneemeyer, F. J. DiSalvo, S. E. Spengler, and J. V. Waszczek, Phys. Rev. B 30, 4297 (1984);

${ }^{25}$ S. Yue, M. Tian, and Y. Zhang, Phys. Lett. A 280, 376 (2001). 
${ }^{26}$ Y. Liu, C. Li, J. Wang, D. Yin, J. Shi, and R. Xiong, Physica B 405, 2857 (2010).

${ }^{27}$ H. Mutka, S. Bouffard, J. Dumas, and C. Schlenker, J. Phys. (Paris) Lett. 45, L729 (1983); H. Mutka, S. Bouffard, M. Sanquer, J. Dumas, and C. Schlenker, Mol. Cryst. Liq. Cryst. 121, 133 (1985).

${ }^{28}$ S. Ravy, S. Rooouziere, J. P. Pouget, S. Brazovskii, J. Marcus, J. F. Berar, and E. Elkaim, Phys. Rev. B 74, 174102 (2006).

${ }^{29}$ S. M. DeLand, G. Mozurkewich, and L. D. Chapman, Phys. Rev. Lett. 66, 2026 (1991).

${ }^{30}$ V. Ya. Pokrovskii and S. V. Zaitsev-Zotov, Phys. Rev. B 61, 13261 (2000); S. V. Zaitsev-Zotov, Microelectronic Engineering 69, 549 (2003).

${ }^{31}$ Detailed description of the experimental setup is given in the supplementary information of Ref. 2.
${ }^{32}$ J. Demsar, K. Biljakovic, and D. Mihailovic, Phys. Rev. Lett. 83, 800 (1999).

${ }^{33}$ K. Shimatake, Y. Toda, and S. Tanda, Phys. Rev. B 73, 153403 (2006).

${ }^{34}$ P. Kusar, V. V. Kabanov, J. Demsar, T. Mertelj, S. Sugai, and D. Mihailovic, Phys. Rev. Lett. 101, 227001 (2008).

${ }^{35}$ M. Beyer, M. Beck, D. Städter, H. Schäfer, V. V. Kabanov, G. Logvenov, I. Bozovic, G. Koren, and J. Demsar, Phys. Rev. B 83, 214515 (2011).

${ }^{36}$ C. Giannetti, G. Zgrablic, C. Consani, A. Crapaldi, D. Nardi, G. Ferrini, G. Dhalenne, A. Revcolevschi, and F. Parmigiani, Phys. Rev. B 80, 235129 (2009).

${ }^{37}$ W. J. Schutte and J. L. de Boer, Acta Cryst. B 49, 579 (1993).

${ }^{38}$ J. Wang, R. Xiong, N. Shu, J. Shi, Z. Jin, J. Mater. Sci. Technol. 23, 5 (2007). 